Revista Brasileira de

Engenharia Agrícola e Ambiental

v. 15 , n.5, p.435-442, 2011

Campina Grande, PB, UAEA/UFCG - http://www.agriambi.com.br

agriambi Protocolo 202.09 - 30/12/2009 • Aprovado em 03/03/2011

\title{
Compressibilidade de um Argissolo Amarelo distrocoeso submetido a diferentes manejos
}

\author{
Veronildo S. O liveira ${ }^{2}$, Mário M. Rolim², Yuri D. J. Costa ${ }^{3}$, \\ Elvira M. R. Pedrosa² \& Ênio F. de F. e Silva²
}

\begin{abstract}
RESU MO
O objetivo deste trabalho foi avaliar a compressibilidade de um Argissolo Amarelo distrocoeso submetido a diferentes sistemas de manejo sob cultivo de cana-de-açúcar. 0 experimento foi realizado em áreas da U sina Triunfo Industrial, AL, onde se avaliaram os manejos: M A - mata nativa; M SI - manejo sem irrigação; $\mathrm{MCl}$ - manejo com irrigação e M CV manejo com fertirrigação (vinhaça). Todas as áreas estudadas, exceto no MA, vêm utilizando o preparo convencional do solo com uso de grades pesadas. As amostras de solo foram coletadas nas profundidades de 0-20 e 20-40 cm. Trincheiras foram abertas nas quatro áreas de manejo em estudo, quando amostras de solo indeformadas foram retiradas com auxílio de aneis metálicos de $6,4 \mathrm{~cm}$ diâmetro e $2,5 \mathrm{~cm}$ de al tura em cada profundidade. A pós coletadas, as amostras foram saturadas e submetidas a sucções de 0,1 a $10 \mathrm{kPa} ; 50,500$ e $1500 \mathrm{kPa}$, levadas à prensa mecânica e real izado o ensai o de compressibilidade. A partir dos dados obti dos determinou-se a tensão de preconsol idação $\left(\sigma_{p}\right)$ das diversas amostras ensaiadas, cujos resultados foram submeti dos à análise de regressão para a variável tensão de preconsolidação, em função da sucção matricial do solo. Em todos os manejos, a profundidade de $0-20 \mathrm{~cm}$ mostrou ser a mais susceptível à compactação em relação à profundidade de $20-40 \mathrm{~cm}$; o manejo M CV alterou o comportamento compressivo do solo em ambas as profundidades $(0-20$ e 20-40 cm) e a capacidade de suporte do solo foi maior em todos os manejos, nas camadas de $20-40 \mathrm{~cm}$.
\end{abstract}

Palavras-chave: compactação, solo, tensão de preconsolidação, aproveitamento de resíduos

\section{Compressibility of an UItisol submitted to different managements}

\begin{abstract}
The objective of this paper was to evaluate the compressibility of an UItisol submitted to different managements of sugarcane cultivation. The investigation was carried out at Triunfo Mill, in the State of Alagoas, Brazil. The testing area was composed by four distinct sub-areas: a preserved forest (NF), an area without irrigation (M W O I), an irrigated area (M W I), and an area with vinasse (M W V). All investigated areas, except the forest, were prepared using conventional equipment for soil preparation. Soil samples were collected within depths ranging from 0 to $20 \mathrm{~cm}, 20$ to $40 \mathrm{~cm}$, and 40 to $60 \mathrm{~cm}$. A trench was dug in the four selected areas and four undeformed samples were collected in each depth. The samples were collected with metallic rings with dimensions of $6.4 \mathrm{~cm}$ in diameter and $2.5 \mathrm{~cm}$ in height. In the laboratory, the samples were previously saturated and submitted to soil suctions raging from 0.1 to $1500 \mathrm{kPa}$ in order to build the soil-water characteristic curves. Confined compression tests were performed with saturated samples. The results obtained from the compression tests were used for the determination of the void ratio (e) and the pre-consolidation stress $\left(\sigma_{p}\right)$ of the tested samples. D ata were submitted to regression analysis and the differences among the obtained means were compared using the test of Tukey at $5 \%$ of probability. Regression analysis for pre-consolidation stress in function of water content $(\mathrm{Ug})$ and correlations among the several variables were carried out at 5-\% significance. Within all investigated areas, the depth range from 0 to $20 \mathrm{~cm}$ showed to be more susceptible to compaction in relation to the depth range from 20 to $40 \mathrm{~cm}$. Management M WV was found to modify the compressive behavior of the soil in both depth ranges. In all investigated areas, the soil bearing capacity was higher in the depth range of 20 to $40 \mathrm{~cm}$.
\end{abstract}

Key words: compaction, soil, pre-consolidation stress, residue use

\footnotetext{
${ }^{1}$ Parte da Tese de doutorado do primeiro autor, UFRPE

2 U FRPE/DTR. Rua D om Manoel de Medeiros s/n, D ois Irmãos, CEP 52171-900, Recife, PE. Fone: (81) 3320-6276. E-mail: verofat@dtr.ufrpe.br; rolim@dtr.ufrpe.br; elvira.pedrosa@dtr.ufrpe.br; enio.silva@dtr.ufrpe.br

3 UFRN/Centro de Tecnologia. E-mail: ydjcosta@ct.ufrn.br
} 


\section{INTRODUÇÃO}

O aumento da intensidade de uso da mecanização agrícola na cultura da cana-de-açúcar, principalmente com as operações de preparo convencional (aração e gradagem) vem causando modificações físico-mecânicas ao solo, com consequente degradação da estrutura, seja promovendo a pulverização dos horizontes superficiais ou a compactação dos horizontes subsuperficiais. Por outro lado, as operações de colheita são as que, a cada ano, mais utilizam máquinas pesadas, conferindo ao solo pressões críticas que favorecem a compactação, principalmente quando utilizadas em condições inadequadas de umidade, sendo esta uma das maiores preocupações para a agricultura moderna.

Segundo Macedo et al. (2010a), quando os solos são submetidos à ação de compressão por máquinas e equipamentos agrícolas, sofrem deformações e compactações cujas intensidades estão relacionadas à sua compressibilidade.

Estudos desenvolvidos por Kondo \& Dias Júnior (1999), têm sido de grande importância no sentido de se avaliar, conjuntamente, a suscetibilidade do solo a compactação e o momento ideal para execução das operações agrícolas.

Além da densidade, o teor de água é um dos fatores que determinam a magnitude da deformação que pode ocorrer no solo, pois age como lubrificante entre as partículas, permitindo o seu deslocamento (Silva et al., 2000).

O acúmulo de matéria orgânica no solo pode aumentar a resistência do solo à compactação ou reduzir seus efeitos; no entanto, a magnitude e o tipo de efeito são dependentes da textura do solo e dos efeitos associados sobre a retenção de água, coesão e densidade do solo (Braida et al., 2010), contudo, são poucas as pesquisas no meio agrícola que relacionem a sucção matricial com índices da mecânica do solo. Alguns estudos sobre modelagem da compactação do solo vêm sendo desenvolvidos utilizando-se técnicas aplicadas em geotecnia, como no caso do ensaio de compressão uniaxial ou triaxial. Neste ensaio são obtidos a tensão de preconsolidação, o índice de compressão, o índice de recompressão, coesão e ângulo de atrito interno do solo (Arvidsson \& Keller, 2004). Poodt et al. (2003) identificaram a coesão e a tensão de preconsolidação como sendo os parâmetros mais importantes na previsão de risco à compactação subsuperficial e são parâmetros extensamente usados em modelos de compactação (Defossez \& Richard, 2002; Pereira et al., 2007).

A tensão de preconsolidação é obtida a partir de uma curva de compressão que representa graficamente a relação entre o logaritmo da tensão vertical aplicada ao solo a parâmetros relacionados com a estrutura do solo, tais como índice de vazios, porosidade ou densidade do solo, obtida a partir de um ensaio de compressão uniaxial (Mosaddeghi et al., 2000.; Macedo et al, 2010b).

A curva de compressão apresenta duas regiões distintas: a primeira, onde ocorrem pequenas deformações elásticas e reversíveis, sendo a região da curva que demonstra um estado do solo onde ocorre o cultivo ou pode ser trafegado, sem que se dê a compactação adicional; outra região corresponde à reta de compressão virgem a partir da qual as tensões aplicadas ao solo resultarão em deformações irreversíveis. A tensão de preconsolidação delimita estas regiões (Lembert \& Horn, 1991; Dias Júnior \& Pierce, 1996; Mosaddeghi et al., 2003).

Segundo Dias Júnior \& Pierce (1996), os modelos matemáticos que se baseiam na tensão de preconsolidação consideram a história da tensão em termos da tensão de preconsolidação como função do teor de água no solo. Neste modelo, a tensão de preconsolidação representa o valor máximo da tensão que poderá ser aplicada ao solo para evitar a compactação adicional. Desta forma, a tensão de preconsolidação do solo expressa, também, a capacidade de suporte de carga dos solos parcialmente saturados (Kondo \& Dias Júnior, 1999; Silva et al., 2006).

Enquanto a tensão de preconsolidação reflete as tensões às quais o solo já foi submetido, o índice de compressão revela a suscetibilidade do solo a compactação (Silva et al., 2000). Quanto maior seu valor, maior também será a inclinação da reta de compressão virgem e mais suscetível o solo será à compactação, pois indica maiores deformações com a carga aplicada (Kondo \& Dias Júnior, 1999).

Uma outra forma de apresentar a capacidade de suporte de carga do solo pode ser utilizando-se as curvas SI (Suction increase) e LC (Loading Collapse), que representam situações de carregamento e de sucção matricial já ocorridos no solo. Segundo Alonso et al. (1987) essas curvas definem duas regiões: uma elástica e outra plástica. Para Costa et al. (2003) as curvas LC e SI definem os limites que podem ser utilizados para estimar a capacidade de suporte de solos não-saturados.

Neste contexto, o presente trabalho teve como objetivo avaliar a suscetibilidade a compactação de um Argissolo Amarelo distrocoeso, em diferentes sistemas de uso e manejo, sob cultivo de cana-de-açúcar, através do comportamento compressivo, identificando sua capacidade de suporte em função da tensão de preconsolidação e da sucção matricial do solo.

\section{Material e mÉtodos}

O experimento foi conduzido em área da Usina Triunfo Agroindustrial, localizada entre os paralelos $9^{\circ} 40^{\prime} 47^{\prime \prime}$ e $9^{\circ} 42^{\prime}$ $30^{\prime \prime} \mathrm{S}$ e nos meridianos $36^{\circ} 08^{\prime} 12^{\prime \prime}$ e $36^{\circ} 05^{\prime} 03^{\prime \prime} \mathrm{W}$, no município da Boca da Mata, AL; o clima da área de estudo, segundo classificação de Köppen, é tropical chuvoso com verão seco; a precipitação média anual fica em torno de $1200 \mathrm{~mm}$; o solo utilizado foi classificado, segundo EMBRAPA (2006), como Argissolo Amarelo distrocoeso, textura média/argilosa, fase floresta subperenifólia e relevo plano.

Escolheram-se quatro situações distintas de uso e manejo do solo: uma coberta com vegetação nativa (floresta subperenifólia) MA - mata e as outras três cultivadas continuamente com cana-de-açúcar, MSI - manejo sem irrigação; MCI - manejo com irrigação; MCV - manejo com fertirrigação (vinhaça). A área sob manejo sem irrigação (sequeiro) há mais de 30 anos, não recebeu nenhuma irrigação ou fertirrigação; na área irrigada foi aplicada uma lâmina de $120 \mathrm{~mm}$ de água, em duas parcelas, durante o ciclo da cultura, através de aspersão por canhão, com vazão de $100 \mathrm{~m}^{3} \mathrm{~h}^{-1}$, há mais de 25 anos; na área com fertirrigação foram aplicados, anualmente, $500 \mathrm{~m}^{3} \mathrm{de}$ 
vinhaça diluídos através de aspersão com $150 \mathrm{~m}^{3} \mathrm{~h}^{-1}$ de vazão, há mais de 25 anos. A caracterização das áreas se encontra na Tabela 1.

Tabela 1. Caracterização do Argissolo Amarelo distrocoeso, em diferentes sistemas de manejo e profundidades sob cultivo de cana-de-açúcar

\begin{tabular}{|c|c|c|c|c|c|}
\hline \multirow{3}{*}{$\begin{array}{c}\text { Camadas } \\
(\mathrm{cm})\end{array}$} & \multicolumn{3}{|c|}{ Granulometria } & \multirow{3}{*}{$\begin{array}{c}\text { Ds } \\
\mathrm{Mg} \mathrm{m}^{-3}\end{array}$} & \multirow{3}{*}{$\begin{array}{l}\text { M.0. } \\
(\%)\end{array}$} \\
\hline & Areia & Silte & Argila & & \\
\hline & \multicolumn{3}{|c|}{$\mathrm{g} \mathrm{kg}^{-1}$} & & \\
\hline \multirow{3}{*}{$\begin{array}{r}0-20 \\
20-40\end{array}$} & & MA & & & \\
\hline & 717,4 & 56,6 & 226,0 & 1,3 & 3,3 \\
\hline & 663,7 & 62,8 & 243,5 & 1,4 & 2,8 \\
\hline \multicolumn{6}{|c|}{ MSI } \\
\hline $0-20$ & 677,4 & 80,3 & 242,2 & 1,48 & 1,7 \\
\hline $20-40$ & 581,2 & 47,8 & 371,0 & 1,6 & 0,9 \\
\hline \multicolumn{6}{|c|}{$\mathrm{MCl}$} \\
\hline $0-20$ & 707,4 & 57,8 & 234,7 & 1,63 & 1,9 \\
\hline $20-40$ & 648,7 & 44,05 & 307,2 & 1,52 & 1,2 \\
\hline \multicolumn{6}{|c|}{ MCV } \\
\hline $0-20$ & 722,4 & 56,5 & 221,0 & 1,53 & 2,6 \\
\hline $20-40$ & 751,2 & 20,3 & 228,5 & 1,55 & 1,8 \\
\hline
\end{tabular}
vinhaça

O preparo do solo das áreas cultivadas foi realizado sempre por ocasião da renovação do canavial o que, normalmente, ocorre após o quarto corte, utilizando-se implementos de preparo convencional: grade pesada, seguida de gradagem leve, precedida de subsolagem com implemento de hastes parabólicas, trabalhando a $40 \mathrm{~cm}$ de profundidade, a fim de quebrar a camada compactada, com posterior sulcagem a 25 $\mathrm{cm}$ de profundidade, sendo a adubação e o plantio realizados manualmente.

As amostras de solo foram coletadas em março de 2006, nas quatro áreas de manejo em estudo, nas camadas de 0-20, 20-40 $\mathrm{cm}$, com quatro repetições. As três áreas estavam cultivadas com cana-de-açúcar, que se encontravam na mesma idade, e cada parcela $(50 \times 50 \mathrm{~m})$ corresponde a um tipo de manejo. Em cada parcela foram sorteados 4 pontos de amostragem, sempre nas entrelinhas da cultura da cana-de-açúcar, retirando-se amostras indeformadas na posição central das duas camadas estudadas.

Amostras indeformadas de cada tratamento e camada amostrada foram utilizadas para determinação da curva característica de retenção de água do solo, sendo utilizado o aparelho de placas porosas (funil de Haynes) para os pontos de baixa tensão, associados aos dados dos pontos de maior tensão obtidos no aparelho de Richards. Assim, amostras saturadas foram submetidas a sucções de 0,1 a $10 \mathrm{kPa}$ (no funil) e 50, 500 e $1500 \mathrm{kPa}$. (na câmara). Os respectivos teores gravimétricos de água foram multiplicados pela densidade do solo de cada camada obtendo-se, então, o teor volumétrico de água e, para a construção da curva de (è, $\mathrm{em} \mathrm{m}^{3} \mathrm{~m}^{-3}$ ) x sucção $\left(\mathrm{u}_{\mathrm{a}}-\mathrm{u}_{\mathrm{w},}, \mathrm{em} \mathrm{kPa}\right)$, conforme (Figura 1).

Para o ensaio de compressibilidade, foram abertas, em cada parcela, 4 trincheiras de onde foram retiradas 4 amostras indeformadas por camada, totalizando 32 amostras por área de manejo e $128 \mathrm{em}$ todo o estudo. As amostras foram obtidas por
A.

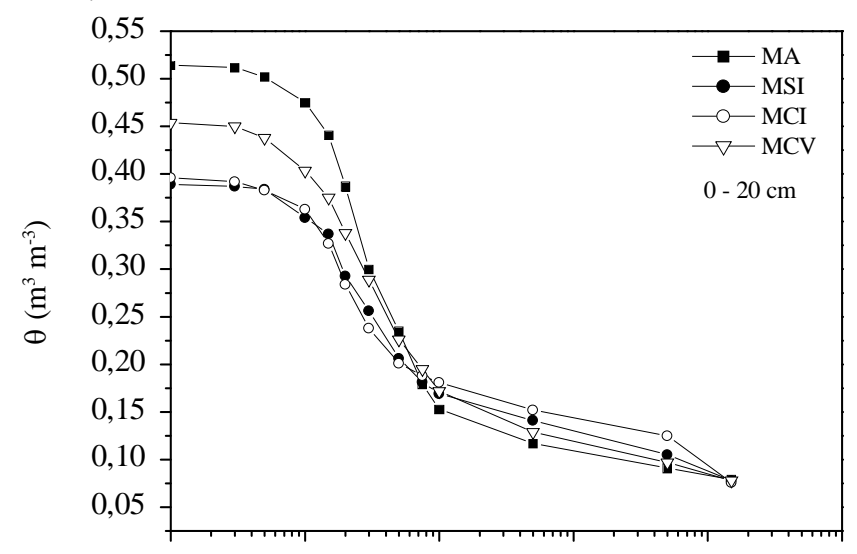

B.

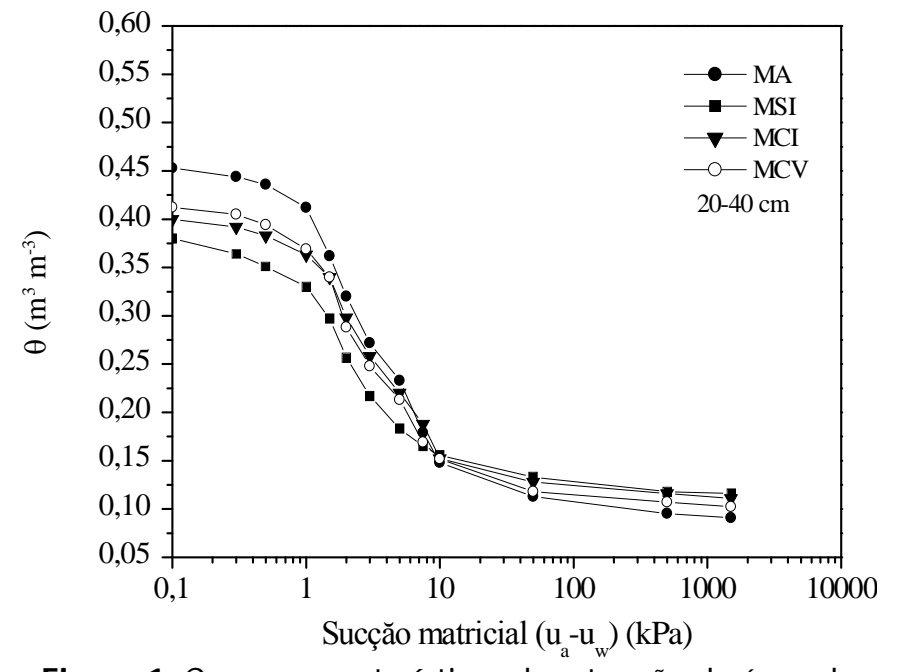

Figura 1. Curvas características de retenção de água de um A rgissolo amarelo distrocoeso sob sistemas de manejo M A - mata; MSI - manejo sem irrigação; $\mathrm{MCI}$ - manejo com irrigação; MCV - manejo com fertirrigação (vinhaça), ajustadas pela equação de van Genuchten, nas profundidades de $0-20$ (A) e $20-40 \mathrm{~cm}$ (B)

um coletor cilíndrico com capacidade para dois aneis metálicos com dimensões de 6,4 × 2,5 cm, no centro das camadas de 0-20 e 20-40 cm, nas respectivas áreas de manejo e uso (MA, MSI, MCI e MCV), e envolvidas com filme plástico e acondicionadas em recipientes de isopor.

No laboratório as amostras para o ensaio de compressibilidade foram devidamente preparadas e saturadas a temperatura ambiente, tendo sido ensaiadas em prensa de adensamento, tipo Bishop, da solotest cuja metodologia segue a norma NBR-12007/90 (ABNT, 1990), com adaptações para solo não saturado, nos seguintes teores de água: 0,08; 0,12; 0,16 e $0,19 \mathrm{~kg} \mathrm{~kg}^{-1}$. O ensaio consistiu na aplicação de pressões estáticas de 25, 50, 100, 200, 400, 800 e $1600 \mathrm{kPa}$, com leituras no defletômetro aos 15 e $30 \mathrm{~s}$. Na prensa a amostra foi submetido a cargas verticais, sendo cada carregamento aplicado em estágio, com duração de cinco minutos, e só se iniciando um novo estágio após cessadas as deformações devidas ao estágio anterior. A partir do ensaio realizado determinaram-se as tensões de preconsolidação. 
Com os dados de sucção matricial obtidos na curva de retenção de água do solo de cada manejo e as respectivas tensões de preconsolidação, obtiveram-se as curvas limites que, segundo Alonso et al. (1987), representam situações de carregamento e de sucção matricial, já ocorridas no solo. Deste modo, as curvas SI (Suction increase) e LC (Loading Collapse) definem duas regiões: uma elástica e outra plástica.

Em investigações de campo Costa et al. (2003) utilizaram as curvas limites representadas por modelo exponencial da seguinte forma:

$$
\sigma_{\mathrm{p}}=\mathrm{a}+\mathrm{b}\left(\mathrm{u}_{\mathrm{a}}-\mathrm{u}_{\mathrm{w}}\right)^{\mathrm{c}}
$$

em que:

$$
\begin{aligned}
& \sigma_{p}-\text { tensão de preconsolidação } \\
& a, \text { b e c - parâmetros de ajuste } \\
& u_{a}-\text { pressão do ar } \\
& u_{w}-\text { pressão da água } \\
& \left(u_{a}-u_{w}\right) \text { - sucção matricial }
\end{aligned}
$$

Os resultados foram submetidos à análise de variância e as médias comparadas pelo teste de Tukey a $5 \%$ de probabilidade. As análises de regressões relativas ao ensaio de compressibilidade foram realizadas segundo os aplicativos SAS (1999) e Origen 6.0 e, para comparações entre as regressões, foi utilizado o procedimento descrito em Snedecor \& Cochran (1989).

\section{RESULTADOS E DisCUSSÃO}

Constatou-se diferença significativa nas comparações entre médias dos manejos de cana-de-açúcar e os teores de água do solo sobre a tensão de preconsolidação do solo (Tabela 2). $\mathrm{O}$ teor de água foi significativo a 5\% na avaliação da tensão de preconsolidação do solo, nos quatro manejos e camadas estudadas. A tensão de preconsolidação aumentou com a

Tabela 2. Valores médios da tensão de preconsolidação $(\mathrm{kPa})$ em função dos sistemas de manejo e teor de água nas camadas de 0-20 e 20-40 cm em um Argissolo Amarelo distrocoeso

\begin{tabular}{cccccc}
\hline Teor de & \multicolumn{5}{c}{ Sistema de manejo } \\
\cline { 2 - 6 } kg kg $^{-1}$ & MA & MSI & MCI & MCV & Média \\
\multicolumn{5}{c}{ Camada de $0-20 \mathrm{~cm}$} \\
0,08 & 113,00 & 160,77 & 157,40 & 127,57 & $139,43 \mathrm{~A}$ \\
0,12 & 83,27 & 112,15 & 112,72 & 88,97 & $99,28 \mathrm{~B}$ \\
0,15 & 72,67 & 84,20 & 83,85 & 72,67 & $78,35 \mathrm{C}$ \\
0,19 & 42,67 & 66,85 & 65,27 & 53,37 & $57,04 \mathrm{D}$ \\
Média & $77,90 \mathrm{~B}$ & $105,99 \mathrm{~A}$ & $104,56 \mathrm{~A}$ & $85,64 \mathrm{~B}$ \\
\hline \multicolumn{5}{c}{ Camada de 20-40 cm } \\
0,08 & $112,65 \mathrm{Ca}$ & $161,88 \mathrm{Aa}$ & $157,10 \mathrm{Aa}$ & $131,27 \mathrm{Ba}$ \\
0,12 & $85,75 \mathrm{Bb}$ & $126,19 \mathrm{Ab}$ & $122,57 \mathrm{Ab}$ & $102,35 \mathrm{Bb}$ \\
0,15 & $75,18 \mathrm{Bb}$ & $94,70 \mathrm{Abc}$ & $94,08 \mathrm{Abc}$ & $87,03 \mathrm{Abb}$ \\
0,19 & $54,92 \mathrm{BC}$ & $78,80 \mathrm{Ac}$ & $74,65 \mathrm{Ac}$ & $77,80 \mathrm{Ab}$ \\
\hline
\end{tabular}

Letras maiúsculas comparam diferenças entre os manejos e minúsculas entre profundidade do solo, a nível de $5 \%$ de probabilidade pelo teste de Tukey. Média de quatro repetições. Manejos: $\mathrm{MA}$ - mata, MSI - manejo sem irrigação, $\mathrm{MCl}$ - manejo com irrigado, MCV - manejo com vinhaça redução do teor de água no solo, nas duas profundidades estudadas. Na camada de $0-20 \mathrm{~cm}$ a tensão de preconsolidação variou de 42,67 a 160,77 $\mathrm{kPa}$, para os diferentes sistemas de manejo. Não houve interação entre os manejos nem os teores e água no solo na camada 0-20 cm de profundidade; entretanto, houve diferença significativa a $5 \%$ entre as médias dos manejos e entre as médias dos teores de água no solo, na camada de 0$20 \mathrm{~cm}$ de profundidade.

Os manejos MSI e MCI apresentaram os maiores valores de tensão de preconsolidação, 105,99 e 104,56 kPa, respectivamente, e estatisticamente iguais entre si. Os sistemas MA e MCV não se diferenciaram entre si, porém, apresentaram valores de tensão de preconsolidação mais baixos do que MSI e MCI. Este comportamento do MCV pode estar associado à manutenção da matéria orgânica aplicada na forma de vinhaça, durante mais de 25 anos.

Para a camada de $20-40 \mathrm{~cm}$, houve diferença significativa entre as médias para a interação manejo x teor de água no solo, com comportamento similar ao da camada de 0-20 cm, com redução da tensão de preconsolidação, em função do acréscimo do teor de água nas amostras ensaiadas, apresentando diferenças significativas entre os teores de água no solo. Especificamente, o manejo MA apresentou os menores valores de tensão de preconsolidação, em todos os teores de água no solo, diferindo estatisticamente dos demais.

O comportamento mecânico do solo em relação à tensão de preconsolidação, também foi verificado por outros autores (Kondo \& Dias Júnior, 1999; Silva et al., 2002; Braida et al., 2006). Apesar da ação antrópica sobre o MCV, o comportamento tensão-deformação foi semelhante ao do MA, fato que reforça a preocupação da adoção de manejos que proporcionem a manutenção e/ou o aumento dos teores de matéria orgânica nos solos cultivados. Com a diminuição do teor de água ocorreu aumento da tensão de preconsolidação e aumento da sua capacidade de suporte, tornando o solo mais resistente às cargas externas pois, quanto mais baixos os teores de água maior a coesão entre as partículas, o que torna a matriz do solo mais resistente às deformações provocadas por forças externas.

Os valores de tensão de preconsolidação para as quatro teores de água nos solos estudados foram estatisticamente diferentes, evidenciando a importância do controle deste fator, quando da utilização de máquinas e implementos agrícolas, tendo em vista que o teor de água ideal para a realização de operações agrícolas deve estar na condição friável, ou seja, abaixo do limite de plasticidade do solo.

Com base nas curvas de compressão, foram obtidas as $\left(\sigma_{\mathrm{p}}\right)$ (Tabela 3) e, em seguida, plotadas de acordo com a sucção matricial $\left(\mathrm{u}_{\mathrm{a}}-\mathrm{u}_{\mathrm{w}}\right)$, segundo a equação de regressão (1), obtendose os coeficientes de ajustes a, b, c e o coeficiente de determinação $\mathrm{R}^{2}$.

Observou-se que os valores "a" da equação de regressão $\sigma_{\mathrm{p}}=\mathrm{a}+\mathrm{b}\left(\mathrm{u}_{\mathrm{a}}-\mathrm{u}_{\mathrm{w}}\right)^{\mathrm{c}}$, para os diferentes sistemas de manejo, variaram entre 23,73 e 66,38 , enquanto os de "b" entre 0,68 e 33,24 . Os coeficientes de determinação foram significativos a $1 \%$ e variaram de 0,92 a 0,99 .

O coeficiente "a" representa, em média, a menor tensão de preconsolidação que o solo já suportou. Neste contexto, o manejo MA apresentou valores substancialmente menores para 
Tabela 3. Coeficientes da equação de regressão, $\sigma_{p}=a$ $+b\left(u_{a}-u_{w}\right) c$, para o Argissolo Amarelo distrocoeso nos diferentes sistemas de manejo, nas camadas de 0-20 e 20-40 $\mathrm{cm}$ de profundidade

\begin{tabular}{|c|c|c|c|c|}
\hline \multirow{2}{*}{$\begin{array}{l}\text { Sistemas } \\
\text { de manejo }\end{array}$} & \multicolumn{4}{|c|}{ Coeficiente $\left(\sigma p=a+b\left(u_{a}-u_{w}\right)^{c}\right)$} \\
\hline & a & b & c & $R^{2}$ \\
\hline \multicolumn{5}{|c|}{ Camada de $0-20 \mathrm{~cm}$} \\
\hline MA & 23,73 & 21,328 & 0,206 & $0,92 * *$ \\
\hline MSI & 55,88 & 8,996 & 0,337 & $0,99 * *$ \\
\hline $\mathrm{MCl}$ & 61,43 & 0,685 & 0,603 & $0,99 * *$ \\
\hline MCV & 38,90 & 15,12 & 0,250 & $0,96 * *$ \\
\hline \multicolumn{5}{|c|}{ Camada de $20-40 \mathrm{~cm}$} \\
\hline MA & 31,47 & 27,340 & 0,150 & $0,92 * *$ \\
\hline MSI & 66,38 & 13,750 & 0,201 & $0,96 * *$ \\
\hline $\mathrm{MCl}$ & 40,53 & 29,420 & 0,188 & $0,98 * *$ \\
\hline MCV & 38,27 & 33,239 & 0,140 & $0,98 * *$ \\
\hline
\end{tabular}

Manejos: MA - mata, MSI - manejo sem irrigação, $\mathrm{MCl}$ - manejo com irrigação, MCV - manejo com vinhaça

** significativos a $1 \%$

o parâmetro nas duas profundidades. O manejo MA não recebeu nenhuma ação antrópica, atuando apenas os efeitos dos ciclos de secagem e umedecimento e carga geostática. Apesar da atuação desses dois fatores, não foram observadas modificações nos atributos do solo que pudessem provocar alterações no comportamento mecânico do solo, nas profundidades estudadas. Comportamento similar foi observado por Assis (2002), em estudo de manejo com diferentes sistemas de preparo do solo, comparados com a condição de mata nativa.

No manejo MCV o valor do parâmetro "a" da equação de regressão $\sigma_{\mathrm{p}}=\mathrm{a}+\mathrm{b}\left(\mathrm{u}_{\mathrm{a}}-\mathrm{u}_{\mathrm{w}}\right)^{\mathrm{c}}$ apresentou a menor alteração em relação ao manejo MA e, praticamente, não foi alterado com a profundidade. A maior susceptibilidade da profundidade de 0$20 \mathrm{~cm}$ pode estar associada aos menores valores de densidade do solo nesta profundidade, devido ao efeito da matéria orgânica.

Para os manejos MSI e MCI, observa-se que os valores do parâmetro "a" da equação de regressão $\sigma_{p}=a+b$. $\left(u_{a}-u_{w}\right)^{c}$, foram mais altos e diferentes nas profundidades estudadas.

Verifica-se que os valores de $\sigma_{\mathrm{p}}$, na profundidade de 20-40 $\mathrm{cm}$, foram maiores que os encontrados na profundidade de 0 $20 \mathrm{~cm}$, evidenciando maior capacidade de suporte e se acentuando à medida em que decresce o teor de água no solo. A maior capacidade de suporte nesta profundidade pode estar associada aos maiores valores da densidade do solo e ao aparecimento do caráter coeso, a partir desta profundidade. $\mathrm{O}$ caráter coeso, segundo Ribeiro (2001), é usado para distinguir horizontes pedogenéticos subsuperficiais adensados, muito resistentes à penetração da faca e muito duro a extremamente duros quando secos, passando a friáveis ou firmes quando úmidos.

$\mathrm{Na}$ Tabela 4 pode-se visualizar um resumo da análise estatística, após linearização das equações de regressão, usando-se o procedimento de Snedecor \& Cochran (1989), obtidas para os diferentes manejos, nas camadas estudadas. Observa-se que as equações foram diferentes estatisticamente entre si, com exceção do MCV, podendo-se inferir que a tensão de preconsolidação se mostrou diferente nas profundidades estudadas.
Tabela 4. Comparação das equações de regressão de compressibilidade entre as profundidades de amostragem, após linearização, através do procedimento de Snedecor \& Cochran (1989) para o Argissolo Amarelo distrocoeso submetido a diferentes sistemas de manejo nas duas camadas amostradas

\begin{tabular}{ccccc}
\hline \multirow{2}{*}{ Camada (cm) } & \multicolumn{4}{c}{ Sistema de manejo } \\
\cline { 2 - 5 } $0-20$ vs $20-40$ & MA & MSI & MCI & MCV \\
\hline MA - Mata; $\mathrm{MSI}$ - Manejo sem irrigação; $\mathrm{MCl}-$ Manejo com irrigação; & $\mathrm{MCV}$ - Manejo com vinhaça
\end{tabular}
${ }^{*} \mathrm{e}^{* *}$ significativos a 5 e $1 \%$ e ns não-significativo

Assis \& Lanças (2005), avaliando a compressibilidade de um Nitossolo Vermelho distrófico sob sistemas de plantio direto, preparo convencional e mata nativa, também encontraram diferenças significativas entre camadas estudadas.

Também foram feitas comparações das equações de regressão entre manejos nas duas camadas $(0-20$ e $20-40 \mathrm{~cm})$ de profundidade (Tabela 5). Observa-se que, na camada de $0-20 \mathrm{~cm}$, não houve diferença estatística entre os manejos Mata e MCV, mostrando que a utilização da vinhaça vem, ao longo dos anos, favorecendo a manutenção das características do solo.

Tabela 5. Comparação das equações de regressão de compressibilidade, após linearização, usando-se o procedimento de Snedecor \& Cochran (1989) para o Argissolo Amarelo distrocoeso nos diferentes sistemas de manejo em amostras coletadas nas profundidades de $0-20$ e $20-40 \mathrm{~cm}$

\begin{tabular}{|c|c|c|c|}
\hline \multicolumn{2}{|c|}{ Tratamento } & \multicolumn{2}{|c|}{$\mathbf{F}$} \\
\hline \multicolumn{4}{|c|}{$0.20 \mathrm{~cm}$} \\
\hline $\mathrm{Ma}$ & $x$ & MSI & $* *$ \\
\hline $\mathrm{Ma}$ & $x$ & $\mathrm{MCl}$ & $* *$ \\
\hline $\mathrm{Ma}$ & $x$ & MCV & ns \\
\hline MSI & $x$ & $\mathrm{MCl}$ & $* *$ \\
\hline MSI & $x$ & $\mathrm{MCV}$ & ns \\
\hline MCV & $x$ & $\mathrm{MCl}$ & $* *$ \\
\hline \multicolumn{4}{|c|}{$20-40 \mathrm{~cm}$} \\
\hline $\mathrm{Ma}$ & $x$ & MSI & $* *$ \\
\hline $\mathrm{Ma}$ & $x$ & $\mathrm{MCl}$ & $* *$ \\
\hline $\mathrm{Ma}$ & $x$ & MCV & $* *$ \\
\hline MSI & $x$ & $\mathrm{MCl}$ & $* *$ \\
\hline MSI & $x$ & MCV & * \\
\hline MCV & $x$ & $\mathrm{MCl}$ & $* *$ \\
\hline
\end{tabular}

MA - Mata; MSI - Manejo sem irrigação; MCl - Manejo com irrigação; MCV - Manejo com vinhaça * e ** significativos a 5 e $1 \%$ e ns não-significativo

Na camada de 20 a $40 \mathrm{~cm}$ de profundidade observou-se que, em todos os sistemas de manejo (Tabela 5), a óp foi diferente, mostrando que a capacidade de suporte de carga é maior nesta camada.

Analisando-se as curvas SI e LC dos quatro manejos (Figura 2), que representam áreas que delimitam zonas de deformações plásticas e elásticas, observa-se que na camada de $0-20 \mathrm{~cm}$ o manejo MA (Figura 2A) foi o que apresentou menor área enquanto nos demais houve incremento da área delimitada pelas duas curvas, evidenciando que o manejo provocou alterações irreversíveis, caracterizando a degradação nessas áreas.

Os manejos MSI e MCI apresentaram os maiores valores para a condição de sucção zero, ou seja, na condição saturada, 


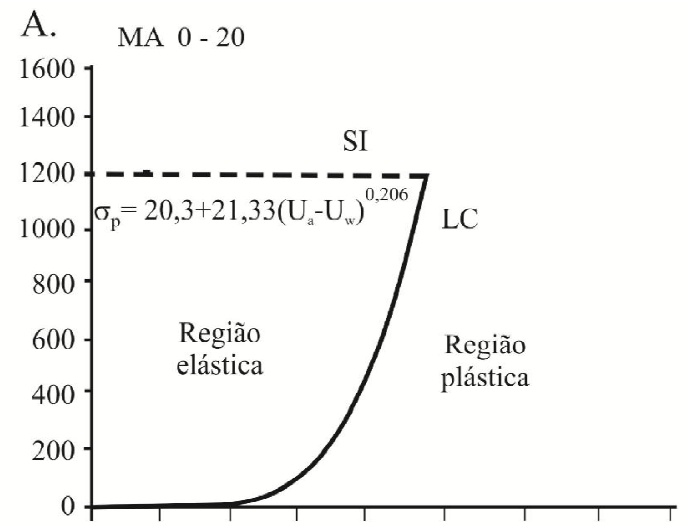

B.

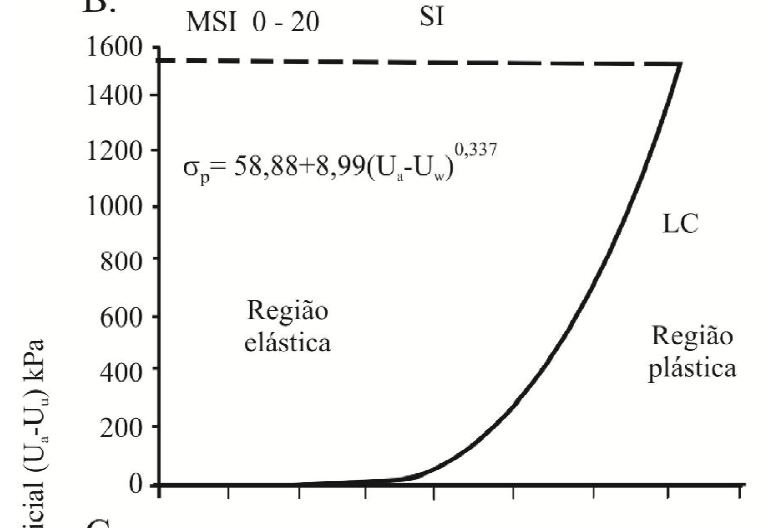

C. MCI $0-20$

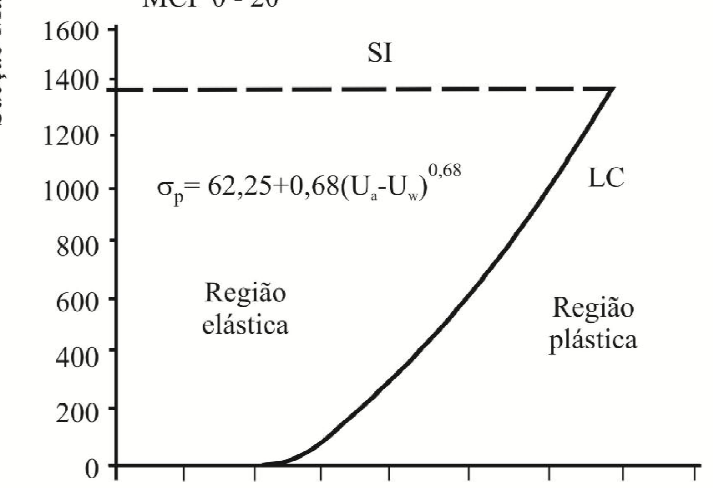

D. MCV $0-20$

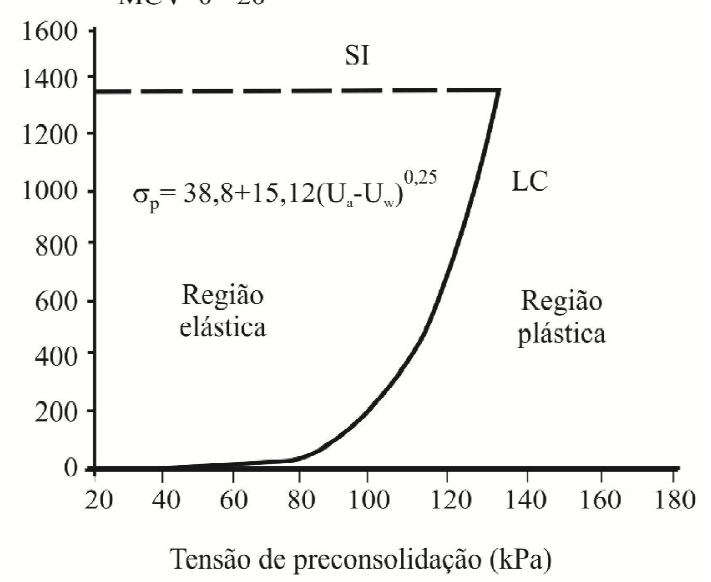

Figura 2. Sucção matricial em função da tensão de preconsolidação dos manejos: A - Mata; B - Manejo sem irrigação; $C$ - manejo com irrigação e D - manejo com vinhaça, na camada $0-20 \mathrm{~cm}$ de profundidade
A.

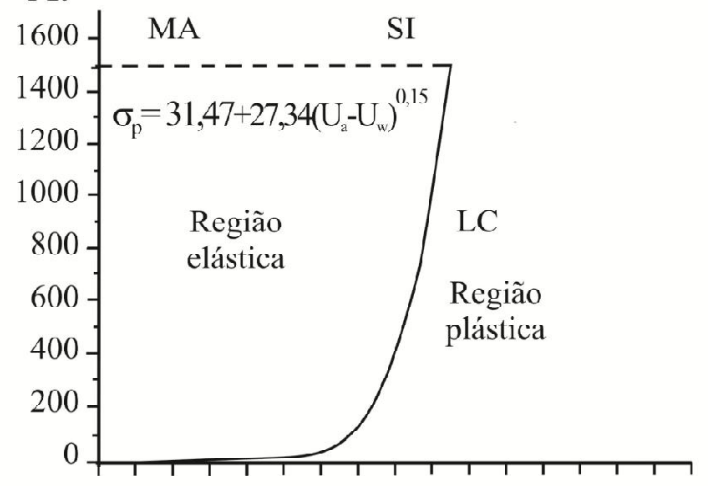

B.

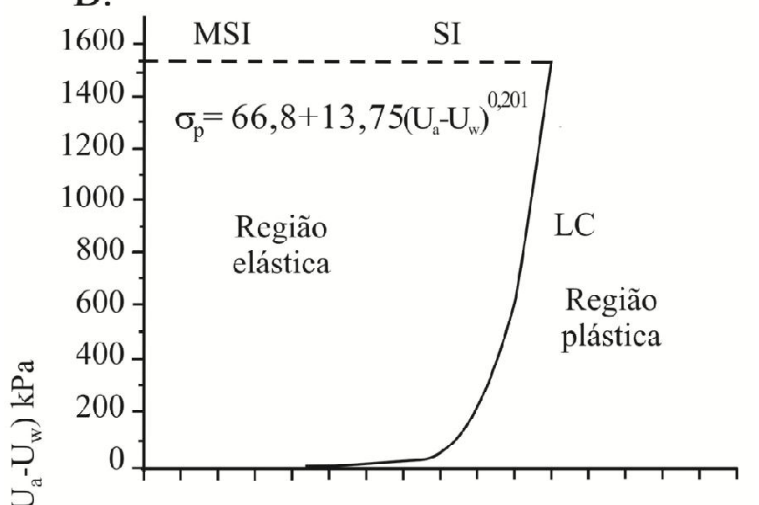

C.

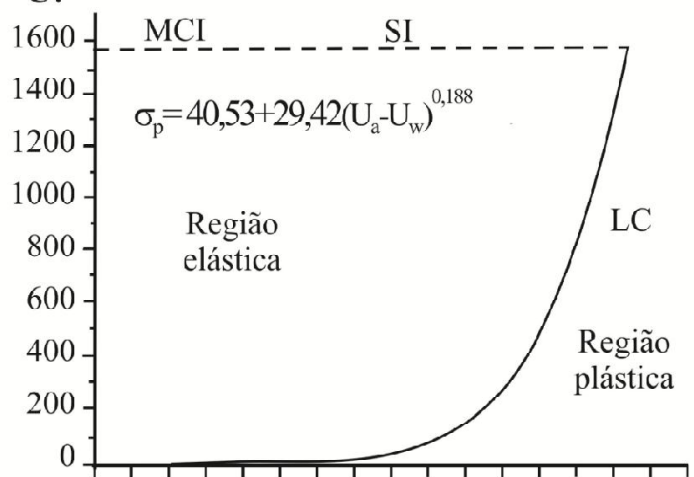

D.

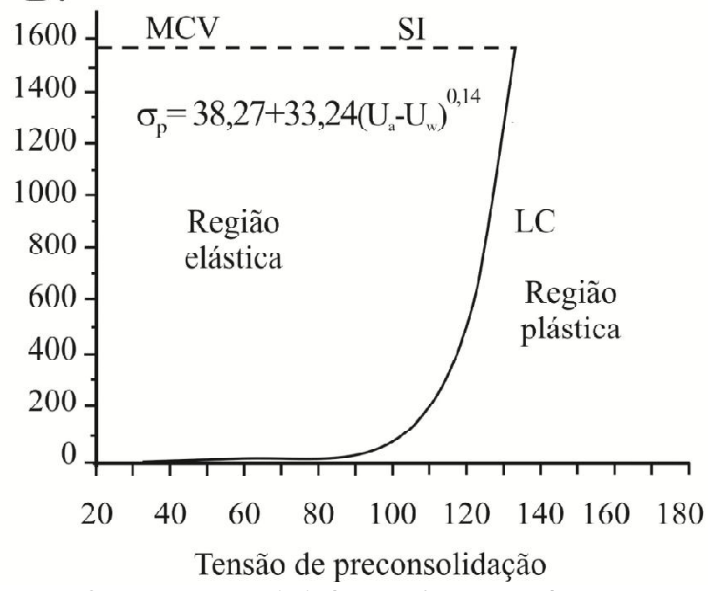

Figura 3. Sucção matricial em função da tensão de preconsolidação dos manejos: A - Mata; B - Manejo sem irrigação; $C$ - manejo com irrigação e $D$ - manejo com vinhaça, na camada $20-40 \mathrm{~cm}$ de profundidade 
58,88 e $62,25 \mathrm{kPa}$, respectivamente, enquanto os manejos MA e MCV os menores foram: 20,3 e 38,9 kPa, respectivamente.

Nos três manejos onde houve cultivo, o solo foi submetido a cargas externas semelhantes (operações agrícolas); entretanto, o manejo MCV (Figura 2D) apresentou uma memória de tensão menor do que os manejos MSI e MCI (Figura 2B e C), devidos, provavelmente, à aplicação continuada de vinhaça durante mais de 25 anos. Embora mais susceptível à compactação, o manejo MCV apresentou menor degradação das propriedades físicas do solo, em virtude da menor tensão de preconsolidação para as umidades estudadas, menor variação da densidade em relação à condição de MA e maior teor de matéria orgânica, corroborando com Silva \& Cabeda (2005), que verificaram que a adição de matéria orgânica na forma de vinhaça contribuiu para os menores valores de tensão de preconsolidação quando comparados com os demais manejos cultivados.

Analisando-se a Figura 3 observa-se, para a profundidade de 20-40 cm, que as curvas SI e LC apresentaram comportamento semelhante aos encontrados na profundidade de $0-20 \mathrm{~cm}$. Os maiores valores na condição de sucção zero ocorreram nos manejos MCI e MSI enquanto os menores ficaram com os manejos MA e MCV.

Considerando-se que os equipamentos utilizados na colheita da cana-de-açúcar (caminhões e carretas) apresentam valores de peso por eixo da ordem de $60 \mathrm{kN}$ e pressão de insuflagem de pneus na ordem de $180 \mathrm{kPa}$ (Kanali et al., 1997) é de se esperar que para as condições em que o solo foi ensaiado, esta operação provocaria compactação adicional ao solo, pois superaria a tensão de preconsolidação e entraria na região plástica, sofrendo deformação irreversível, dependendo da sucção matricial encontrada no campo, no momento de entrada do maquinário no talhão. Assim, as curvas LS e SI podem auxiliar na prevenção do risco de compactação adicional e no monitoramento do tráfego de maquinário podendo, também, subsidiar a escolha das máquinas agrícolas a veículos de transbordo a serem utilizados.

\section{CONCLusÕes}

1. Em todos os tratamentos a profundidade de $0-20 \mathrm{~cm}$, mostrou ser a mais susceptível à compactação em relação à profundidade de $20-40 \mathrm{~cm}$.

2. O manejo MCV proporcionou comportamento compressivo do solo em ambas as camadas (0-20 e 20-40 cm) mais próximo da condição natural.

3. A capacidade de suporte do solo foi maior em todos os manejos nas camadas de $20-40 \mathrm{~cm}$ e menor na de $0-20 \mathrm{~cm}$, obedecendo à seguinte ordem crescente $\mathrm{MCI}>\mathrm{MSI}>\mathrm{MCV}>$ MA.

4. Os manejos MSI e MCI apresentaram maiores áreas limitadas pelas curvas SI e LC, indicando que a compactação do solo influenciou decisivamente a capacidade de suporte de carga do solo.

\section{AgRAdeCIMENTOS}

Os autores agradecem à Usina Triunfo, na pessoa do Engenheiro Agrônomo Arnaldo Jugurta, pelo apoio logístico nas atividades de campo.

\section{LITERATURA CITADA}

ABNT - Associação Brasileira de Normas Técnicas. NBR 12007: Solo. Ensaio de adensamento unidimensional. Rio de Janeiro; ABNT, 1990. 13p.

Alonso, E. E.; Gens, A.; Hight, D. W. General report. Special problem soils. In Proceedings of the 9th European Conference on Soil Mechanics and Foundation Engineering, v.3, p.1087-1146, 1987.

Arvidsson, J.; Keller, T. Soil precompression stress I. A survey of Swedish arable soils. Soil \& Tillage Research, v.77, p.8595, 2004.

Assis, R. L. de. Avaliação dos atributos físicos e da compressibilidade de um Nitossolo Vermelho distroférrico sob sistemas de plantio direto, preparo convencional e mata nativa. Botucatu: FCA/UNESP, 2002. 128p. Tese Doutorado

Assis, R. L.; Lanças, K. P. Avaliação da compressibilidade de um Nitossolo Vermelho distroférrico sob Sistema plantio direto, preparo convencional e mata nativa. Revista Brasileira de Ciência do Solo, v.29, p.509-514, 2005.

Braida, J. A.; Reichardt, J. M.; Veiga, M.; Reinert, D. J. Resíduos vegetais na superfície e carbono orgânico do solo e suas relações com a densidade máxima obtida no ensaio proctor. Revista Brasileira de Ciência do Solo, v.30, p.605-614, 2006.

Braida, J. A.; Reichert, J. M.; Reinert, D. J.; Veiga, M. Teor de carbono orgânico e a susceptibilidade à compactação de um Nitossolo e um Argissolo. Revista Brasileira de Engenharia Agrícola e Ambiental, v. 14, p.131-139, 2010.

Costa, Y. D.; Cintra, J. C.; Zornberg, J. G. Influence of matric suction on the results of plate load tests performed on a Lateritic soil deposit. Geotechnical Testing Journal, v.26, p.1-9, 2003.

Defossez, P.; Richard, G. Models of soil compaction due to traffic and their evaluation. Soil \& Tillage Research, v.67, p.41-64, 2002.

Dias Júnior, M. S.; Pierce, F. J. O processo de compactação do solo e sua modelagem. Revista Brasileira de Ciência do Solo, v.20, p.175-182, 1996.

EMBRAPA - Empresa Brasileira de Pesquisa Agropecuária Centro Nacional de Pesquisa de Solos. Sistema brasileiro de classificação de solos. 2.ed. Rio de Janeiro: EMBRAPA, 2006. 306p.

Kanali, C. L.; Kaumbutho, P. G.; Maende, C. M.; Kamau, J. The use of soil compaction levels in the selection of the fieldsafe sugarcane transport vehicles. Journal of Terramechanics, v.34, p.127-140, 1997.

Kondo, M. K.; Dias Júnior, M. S. Efeito do manejo e da umidade no comportamento compressivo de três Latossolos. Revista Brasileira de Ciências do Solo, v.23, p.497-506, 1999.

Lembert, M.; Horn, R. A method to predict the mechanical strength of agricultural soils. Soil \& Tillage Research, v.19, p.275-286, 1991. 
Macedo, V. R. M.; Silva, A. J. N.; Cabeda, M. S. V. Compressibilidade de um Argissolo Vermelho fisicamente degradado e recuperado. Revista Brasileira de Engenharia Agrícola e Ambiental, v.14, p.812-818, 2010a.

Macedo, V. R. M.; Silva, A. J. N.; Cabeda, M. S. V. Influência de tensões compressivas na pressão de precompactação e no índice de compressão do solo. Revista Brasileira de Engenharia Agrícola e Ambiental, v.14, p.856-862, 2010 b.

Mosaddeghi, M. R.; Hajabbas, M. A.; Hemmat, A.; Afyuni, M. Soil compactibility as affected by soil moisture content and farmyard manure in central Iran. Soil \& Tillage Research, v.55, p.87-97, 2000.

Mosaddeghi, M. R.; Hemmat, A.; Hajabbasi, M. A.; Alexandrou, A. Pre-compression stress and its relation with the physical and mechanical properties of a structurally unstable soil in Central Iran. Soil \& Tillage Research, v.70, p.53-64, 2003.

Pereira, J. O.; Defossez, P. I.; Richards, G. Soil susceptibility to compaction by wheeling as a function of some properties of a silty soil as affected by the tillage system. European Journal of Soil Science, v.58, p.34-44, 2007.

Poodt, M. P.; Koolen, A. J.; van der Linden, J. P. FEM analysis of subsoil reaction on heavy wheel loads with emphasis on soil preconsolidation stress and cohesion. Soil \& Tillage Research, v.73, p.67-76, 2003.

Ribeiro, M. R. Caracterização morfológica dos horizontes coesos dos solos dos tabuleiros costeiros. In: Workshop: Coesão em Solos dos Tabuleiros Costeiros, 2001. Aracaju. Anais... Aracaju: Embrapa Tabuleiros Costeiros, 2001. p.165-168.
SAS Institute. SAS/STAT User's Guide, Version 8. Cary: SAS Institute, 1999. 3365p.

Silva, A. J. N.; Cabeda, M. S. V. Compressibilidade de um Argissolo Amarelo de tabuleiro costeiro submetido a pressões uniaxiais. In: Congresso Brasileiro de Ciência do Solo, 30, 2005. Recife. Anais... Viçosa, Sociedade Brasileira de Ciência do Solo, 2005. CD-Room

Silva, A. R.; Dias Júnior, M. S.; Guimarães, P. T. G.; Araújo Júnior, C. F. Modelagem da capacidade de suporte de carga e quantificação dos efeitos das operações mecanizadas em um Latossolo Amarelo cultivado com cafeeiros. Revista Brasileira de Ciência do Solo, v.30, p.207-216, 2006.

Silva, V. R.; Reinert, D. J.; Reichert, J. M. Fatores controladores da compressibilidade de um Argissolo Vermelho-Amarelo distrófico arênico e de um Latossolo Vermelho distrófico típico. II - grau de saturação em água. Revista Brasileira de Ciência do Solo, v.26, p.9-15, 2002.

Silva, V. R.; Reinert, D. J.; Reichert, J. M. Susceptibilidade à compactação de um Latossolo Vermelho Escuro e de um Podzólico Vermelho Amarelo. Revista Brasileira de Ciência do Solo, v.24, p.239-349, 2000.

Snedecor, G. W.; Cochran, W. G. Statistical methods. 8.ed. Ames: Iowa State University Press, 1989. 503p.

van Genuchten, M. T. A closed-form equation predicting the hydraulic conductivity of unsaturated soils. Soil Science Society of American Journal, v.44, p.892-898, 1980. 